\title{
Bayer Corneal Epitheliopathy Grade 4
}

National Cancer Institute

\section{Source}

National Cancer Institute. Bayer Corneal Epitheliopathy Grade 4. NCI Thesaurus. Code C128444.

Corneal ulcer more severe than grade 3. 